\title{
COHOMOLOGICAL CHARACTERIZATION OF THE HILBERT SYMBOL OVER $\mathbb{Q}_{p}^{*}$
}

\author{
FERNANDO PABLOS ROMO
}

(Received 11 February 2004; revised 15 November 2004)

Communicated by K. F. Lai

\begin{abstract}
The aim of this work is to offer a new characterization of the Hilbert symbol over $\mathbb{Q}_{p}^{*}$ from the commutator of a certain central extension of groups. We obtain a characterization for $\mathbb{Q}_{p}^{*}(p \neq 2)$ and a different one for $\mathbb{Q}_{2}^{*}$.
\end{abstract}

2000 Mathematics subject classification: primary 19F15, $20 \mathrm{~F} 12$.

\section{Introduction}

In recent years, characterizations of algebraic symbols have been obtained from the properties of infinite-dimensional vector spaces in order to provide new interpretations for these symbols and to deduce standard theorems from the new definitions in an easy way.

Thus, in 1968 Tate [7] gave a definition of the residues of differentials on curves in terms of traces of certain linear operators on infinite-dimensional vector spaces.

A few years later, in 1989, Arbarello, de Concini and Kac [1] obtained a definition of the tame symbol of an algebraic curve from the commutator of a certain central extension of group. More recently, the author has given an interpretation of this central extension in terms of determinants associated with infinite-dimensional vector subspaces [3], and has defined the Parshin symbol on a surface as iterated tame symbols [4].

This work is partially supported by the DGESYC research contract no. BFM2003-00078 and Castilla y León Regional Government contract SA071/04.

(C) 2005 Australian Mathematical Society $1446-7887 / 05 \$ A 2.00+0.00$ 
In all articles referred to above, the respective reciprocity laws (in particular, the residue theorem) are deduced directly from the finiteness of the cohomology groups $H^{0}\left(C, \mathscr{O}_{C}\right)$ and $H^{1}\left(C, \mathscr{O}_{C}\right)$.

The purpose of the present work is to give a new characterization of the Hilbert symbol over $\mathbb{Q}_{p}^{*}$ by using the method described in [1] and [3]. This definition, which involves topics of Steinberg symbols, allows us to use the results of $[1,2,3]$ to study the properties of this symbol. A remaining problem is to obtain a new proof of the Gauss Reciprocity Law from the statements of this characterization.

Similarly to the computation of the symbol ([6, page 20]), we obtain a characterization for $\mathbb{Q}_{p}^{*}$ with $p \neq 2$ and a different one for $\mathbb{Q}_{2}^{*}$.

For a detailed study of p-adic fields and the Hilbert symbol, the reader is referred to [6].

\section{Preliminaries}

This section is added for the sake of completeness.

2.1. Definition of the Hilbert symbol If $k$ denotes either the field $\mathbb{R}$ of real numbers or the field $\mathbb{Q}_{p}$ of $p$-adic numbers ( $p$ being a prime number), Serre [6] defines the Hilbert symbol $(\cdot, \cdot)_{k}: k^{*} \times k^{*} \rightarrow \mu_{2}$ as:

$$
(a, b)_{k}= \begin{cases}1 & \text { if } z^{2}-a x^{2}-b y^{2}=0 \text { has a non-trivial solution in } k^{3} \\ -1 & \text { otherwise }\end{cases}
$$

where $a, b \in k^{*}$ and $\mu_{2}=\{1,-1\}$.

The Hilbert symbol is a Steinberg symbol ([2, page 94]) because it is bimultiplicative and satisfies $(a, 1-a)_{k}=1$. Moreover, $(a,-a)_{k}=1$ and $(a, b)_{k}=(b, a)_{k}$.

If $k=\mathbb{Q}_{p}$, we shall write $(a, b)_{p}=(a, b)_{k}$.

It is known that $\mathbb{Q}_{p}^{*} \simeq \mathbb{Z} \times \mathscr{U}^{p}$, where $\mathscr{U}^{p}$ is the group of $\mathrm{p}$-adic units. Hence, if $v_{p}$ denotes the $p$-adic valuation, each element $a \in \mathbb{Q}_{p}^{*}$ can be written uniquely in the form $a=p^{\alpha} u$, with $\alpha=v_{p}(a)$ and $u \in \mathscr{U}^{p}$.

Moreover, if we denote by $\mathscr{U}^{2}$ the group of units of $\mathbb{Z}_{2}$, we can define the morphisms of groups $\epsilon, \omega: \mathscr{U}^{2} \rightarrow \mathbb{Z} / 2$ as follows:

$$
\begin{gathered}
\epsilon(u)=\frac{u-1}{2} \quad(\bmod 2)=\left\{\begin{array}{lll}
0 & \text { if } u \equiv 1 \quad(\bmod 4) ; \\
1 & \text { if } u \equiv-1 \quad(\bmod 4),
\end{array}\right. \\
\omega(u)=\frac{u^{2}-1}{8} \quad(\bmod 2)=\left\{\begin{array}{lll}
0 & \text { if } u \equiv \pm 1 \quad(\bmod 8) \\
1 & \text { if } u \equiv \pm 5 & (\bmod 8)
\end{array}\right.
\end{gathered}
$$


Setting $\mathscr{U}_{n}^{p}=1+p^{n} \mathbb{Z}_{p}, \epsilon$ and $\omega$ determine isomorphisms of groups

$$
\epsilon: \mathscr{U}^{2} / \mathscr{U}_{2}^{2} \simeq \mathbb{Z} / 2 \text { and } \omega: \mathscr{U}_{2}^{2} / \mathscr{U}_{3}^{2} \simeq \mathbb{Z} / 2 .
$$

Furthermore, $\mathbb{Q}_{2}^{*} /\left(\mathbb{Q}_{2}^{*}\right)^{2} \simeq \mathbb{Z} / 2 \times \mathbb{Z} / 2 \times \mathbb{Z} / 2$ and each element $a=2^{\alpha} u \in \mathbb{Q}_{2}^{*}$ can be written in the form $(-1)^{\epsilon(u)} 2^{\alpha} 5^{\omega(u)} \bar{u}$, where $\bar{u} \in\left(\mathbb{Q}_{2}^{*}\right)^{2}$.

There exist also isomorphisms of groups $\phi: \mathscr{U}^{p} / \mathscr{U}_{1}^{p} \cong(\mathbb{Z} / p)^{*}$, and if $f \in \mathscr{U}^{p}$, we shall write $f(p)=\phi(\bar{f}) \in(\mathbb{Z} / p)^{*}$.

Thus, given $a, b \in \mathbb{Q}_{p}^{*}, a=p^{\alpha} u$ and $b=p^{\beta} v$, the value of the Hilbert symbol is:

$$
\begin{aligned}
& (a, b)_{p}=(-1)^{\alpha \beta \epsilon(p)}\left(\frac{u^{\beta}}{v^{\alpha}}(p)\right)^{(p-1) / 2} \quad \text { if } p \neq 2, \\
& (a, b)_{2}=(-1)^{\epsilon(u) \epsilon(v)+\alpha \omega(v)+\beta \omega(u)} .
\end{aligned}
$$

2.2. The group $\operatorname{Gl}(V, A)$ and its canonical central extension Let $V$ be a vector space over a field $k$ (in general infinite-dimensional) and let $A$ be a vector subspace of $V$. With the same notation as in [1] we set

$$
\operatorname{Gl}(V, A)=\{f \in \operatorname{Aut}(V) \text { such that } f A \sim A\} \text {, }
$$

where $f \sim f A$ when $\operatorname{dim}_{k}(A+f A / A \cap f A)<\infty$, which is the definition of commensurable subspaces of Tate [7].

If $f \in \mathrm{Gl}(V, A)$, we set $(A \mid f A)=\Lambda(A / A \cap f A)^{*} \bigotimes_{k} \Lambda(f A / A \cap f A), \Lambda$ being the maximal exterior power. Canonically, Arbarello, de Concini and Kac [1] defined a group $\widetilde{G l}(V, A)=\{(f, s)$ with $f \in \mathrm{Gl}(V, A)$ and $s \in(A \mid f A), s \neq 0\}$, which induces a central extension:

$$
1 \rightarrow k^{*} \rightarrow \widetilde{\mathrm{Gl}}(V, A) \rightarrow \mathrm{Gl}(V, A) \rightarrow 1 .
$$

Let us set $A=k[[t]]$ and $V=k((t))$. Since $k((t))^{*} \subseteq \mathrm{Gl}(V, A)$, if we denote by $\{\cdot, \cdot\}_{A}$ the commutator of the above extension and we consider two elements, $f, g \in k((t))^{*}$ with $f=\lambda t^{\alpha}\left(1+\sum_{i \geq 1} a_{i} t^{i}\right)$, and $g=\mu t^{\beta}\left(1+\sum_{j \geq 1} b_{j} t^{j}\right)$, where $\lambda, \beta \in k^{*}$ and $\alpha, \beta \in \mathbb{Z}$, we have that

$$
\{f, g\}_{A}=\frac{\lambda^{\beta}}{\mu^{\alpha}} \in k^{*}
$$

This computation of the commutator can also be found in [3].

2.3. Steinberg symbols For any field $F$, a bimultiplicative mapping $c: F^{*} \times F^{*} \rightarrow A$ to an abelian group, satisfying $c(x, 1-x)=1$ for $x \neq 1$, is called a 'Steinberg symbol' on the field $F$. 
If $F_{v}$ is a discrete valuation field, $\mathscr{O}_{v}$ is the valuation ring, $\mathrm{m}_{v}$ is the unique maximal ideal and $k(v)=\mathscr{O}_{v} / \mathrm{m}_{v}$ is the residue class field, the tame symbol

$$
\begin{aligned}
d_{v}: F_{v}^{*} \times F_{v}^{*} & \rightarrow k(v)^{*} \\
(x, y) & \mapsto(-1)^{v(x) v(y)} \frac{x^{v(y)}}{y^{v(x)}}\left(\bmod \mathfrak{m}_{v}\right)
\end{aligned}
$$

is an easy example of a Steinberg symbol on $F_{v}^{*}$.

For a detailed study of Steinberg symbols, we refer the reader to [2].

\section{Characterization of the Hilbert symbol}

Let us now consider $k=\mathbb{Q}_{p}, A_{p}=\mathbb{Q}_{p}[[t]]$ and $V_{p}=\mathbb{Q}_{p}((t))$.

Since each element $f \in \mathbb{Q}_{p}^{*}$ can be written uniquely in the form $a=p^{v_{p}(f)} u$ with $u \in \mathscr{U}^{p}$, we can consider the injective group morphism

$$
\begin{aligned}
& \varphi: \mathbb{Q}_{p}^{*} \hookrightarrow \mathbb{Q}_{p}((t))^{*}, \\
& p^{\alpha} u \mapsto u t^{\alpha},
\end{aligned}
$$

and we deduce that $\mathbb{Q}_{p}^{*}$ is a commutative subgroup of $\mathrm{Gl}\left(V_{p}, A_{p}\right)$ by considering the homotheties $h_{\varphi(f)}$. Thus the commutator of the following central extension of groups

$$
\mathrm{l} \rightarrow \mathbb{Q}_{p}^{*} \rightarrow \widetilde{\mathrm{Gl}}\left(V_{p}, A_{p}\right) \rightarrow \mathrm{Gl}\left(V_{p}, A_{p}\right) \rightarrow 1
$$

determines a 2-cocycle $\{\cdot, \cdot\}_{p}: \mathbb{Q}_{p}^{*} \times \mathbb{Q}_{p}^{*} \rightarrow \mathscr{U}^{p}$ whose value is $\{f, g\}_{p}=u^{\beta} / v^{\alpha}$, where $f=p^{\alpha} u$ and $g=p^{\beta} v$.

3.1. Hilbert symbol over $\mathbb{Q}_{p}^{*}(\boldsymbol{p} \neq 2) \quad$ From the morphism of groups $\psi_{p}: \mathscr{U}^{p} \rightarrow \mu_{2}$ defined as $\psi_{p}(u)=(u(p))^{(p-1) / 2}$ we have a 2-cocycle $\widetilde{r \cdot, \cdot\}_{p}}: \mathbb{Q}_{p}^{*} \times \mathbb{Q}_{p}^{*} \rightarrow \mu_{2}$, whose value is

$$
\widetilde{\{f, g\}_{p}}=\psi_{p}\left(\{f, g\}_{p}\right)=\left(\frac{u^{\beta}}{v^{\alpha}}(p)\right)^{(p-1) / 2} .
$$

In general, one has that $\widetilde{\left.r_{,} \cdot\right\}_{p}}$ is not a Steinberg symbol because

$$
\left\{p^{-1}, \widetilde{1-p^{-1}}\right\}_{p}=-1
$$

when $p \equiv 3(\bmod 4)$.

We shall now give a cohomological definition of the Hilbert symbol as a distinguished element in the cohomology class $\left[\widetilde{\{\cdot, \cdot\}_{p}}\right] \in H^{2}\left(\mathbb{Q}_{p}^{*}, \mu_{2}\right)$, where $H^{2}(A, B)$ is the group of classes of 2-cocycles $f: A \times A \rightarrow B$ (mod 2-coboundaries) [5]. 
LEMMA 3.1. For each $a \in \mathbb{Z}$, there exists a unique 2-coboundary $c_{a}: \mathbb{Z} \times \mathbb{Z} \rightarrow \mu_{2}$ satisfying the conditions:

- $c_{a}(\alpha, \beta+\gamma)=c_{a}(\alpha, \beta) c_{a}(\alpha, \gamma)$

- $c_{a}(\alpha, \alpha)=(-1)^{\alpha a}$

for $\alpha, \beta, \gamma \in \mathbb{Z}$.

PROOF. Recall that a 2-cocycle $c_{a}: \mathbb{Z} \times \mathbb{Z} \rightarrow \mu_{2}$ is a 2-coboundary when there exists a map $\phi: \mathbb{Z} \rightarrow \mu_{2}$ such that $c_{a}(\alpha, \beta)=\phi(\alpha+\beta) \phi(\alpha)^{-1} \phi(\beta)^{-1}$. Let $\phi(\alpha)=\lambda_{\alpha} \in \mu_{2}$. It follows from the conditions of the lemma that

$$
\lambda_{\alpha}=(-1)^{\alpha(\alpha-1) a / 2} \lambda_{1}^{\alpha} \quad \text { for each } \alpha \in \mathbb{Z} .
$$

Hence $c_{a}(\alpha, \beta)=(-1)^{\alpha \beta a}$ is the unique 2-coboundary that satisfies the statement of the lemma.

THEOREM 3.2. There exists a unique Steinberg symbol $(\cdot, \cdot)_{p}$ in the cohomology class $\left[\widetilde{\cdot, \cdot \cdot\}_{p}}\right] \in H^{2}\left(\mathbb{Q}_{p}^{*}, \mu_{2}\right)$ satisfying the condition

$$
(f, g)_{p}=\widetilde{\{f, g\}_{p}} \text { if } v_{p}(f)=0 .
$$

This Steinberg symbol is the Hilbert symbol over $\mathbb{Q}_{p}^{*}$.

PROOF. Let $v(f, g)=c^{\prime}(f, g) \widetilde{\{f, g\}_{p}}$ be a Steinberg symbol in the cohomology class $\left[\widetilde{\{\cdot \cdot\}_{p}}\right] \in H^{2}\left(\mathbb{Q}_{p}^{*}, \mu_{2}\right)$ such that $c^{\prime}(f, g)=1$ for $v_{p}(f)=0$. Since $c^{\prime}$ is a 2-coboundary, one has that $c^{\prime}(f, g)=1$ when $v_{p}(g)=0$ and, therefore, there exists a commutative diagram

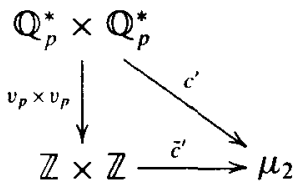

where $\tilde{c}^{\prime}$ is a 2-coboundary satisfying $\tilde{c}^{\prime}(\alpha, \beta+\gamma)=\tilde{c}^{\prime}(\alpha, \beta) \tilde{c}^{\prime}(\alpha, \gamma)$.

Furthermore, since $v(f,-f)=1$ for all $f \in \mathbb{Q}_{p}^{*}$, one has that $\tilde{c}^{\prime}(\alpha, \alpha)=$ $(-1)^{\alpha(p-1) / 2}=(-1)^{\alpha \epsilon(p)}$. It then follows from Lemma 3.1 that $\tilde{c}^{\prime}(\alpha, \beta)=(-1)^{\alpha \beta \epsilon(p)}$ and $c^{\prime}(f, g)=(-1)^{v_{p}(f) v_{p}(g) \epsilon(p)}$.

Thus, the unique Steinberg symbol in $\left[\widetilde{\cdot, \cdot\}_{p}}\right] \in H^{2}\left(\mathbb{Q}_{p}^{*}, \mu_{2}\right)$ is

$$
v(f, g)=(-1)^{v_{p}(f) v_{p}(g) \epsilon(p)} \widetilde{[f, g\}_{p}},
$$

which is the Hilbert symbol. 
REMARK 3.3. The above property, which characterizes the Hilbert symbol in $\mathbb{Q}_{p}^{*}$ is equivalent to one of the conditions that Serre gave to define local symbols on algebraic curves ([5]) which are also Steinberg symbols.

Let us now consider in $\mathbb{Q}_{p}^{*}$ the structure of the topological group induced by the $p$-adic valuation and let us consider $\mu_{2}$ as a topological group with the discrete topology.

CONJECTURE 3.4. $(\cdot, \cdot)_{p}$ is the unique continuous Steinberg symbol in the cohomology class $\left[\{\cdot \cdot \cdot\}_{p}\right] \in H^{2}\left(\mathbb{Q}_{p}^{*}, \mu_{2}\right)$.

3.2. Hilbert symbol over $\mathbb{Q}_{2}^{*} \quad$ Let us consider the morphism of groups $\psi_{2}: \mathscr{U}^{2} \rightarrow \mu_{2}$ defined as $\psi_{2}(u)=(-1)^{\omega(u)}$. This map induces a 2-cocycle $\{\cdot \cdot \cdot\}_{2}: \mathbb{Q}_{2}^{*} \times \mathbb{Q}_{2}^{*} \rightarrow \mu_{2}$ whose value is

$$
\widetilde{\{f, g\}_{2}}=\psi_{2}\left(\{f, g\}_{2}\right)=(-1)^{\beta \omega(u)+\alpha \omega(v)} \text {, }
$$

where $f=2^{\alpha} u$ and $g=2^{\beta} v$.

Again, $\left\{\widetilde{r, \cdot\}_{2}} \text { is not a Steinberg symbol because } \widetilde{[6,-5}\right\}_{2}=-1$. We shall now determine the relation between the commutator $\{\cdot, \cdot\}_{2}$ and the Steinberg symbol $(\cdot, \cdot)_{2}$ in the group of 2-cocycles $Z^{2}\left(\mathbb{Q}_{2}^{*}, \mu_{2}\right)$.

THEOREM 3.5. There exists a unique 2-cocycle $c_{2} \in Z^{2}\left(\mathbb{Q}_{2}^{*}, \mu_{2}\right)$ such that $c_{2} \tilde{r,} \cdot l_{2}$ is a non-trivial Steinberg symbol. This symbol is the Hilbert symbol $(\cdot, \cdot)_{2}$. Moreover, $c_{2}$ is not a 2-coboundary and hence $(\cdot, \cdot)_{2} \notin\left[\left\{\widetilde{\cdot \cdot \cdot\}_{2}}\right] \in H^{2}\left(\mathbb{Q}_{2}^{*}, \mu_{2}\right)\right.$.

ProOF. Let $c_{2} \in Z^{2}\left(\mathbb{Q}_{2}^{*}, \mu_{2}\right)$ such that $v=c_{2}\left\{\widetilde{r \cdot \gamma_{2}}\right.$ is a Steinberg symbol. Since $\widetilde{\left.r_{,},\right\}_{2}}$ is a bimultiplicative map, $c_{2}$ must be bimultiplicative and one has that

$$
c_{2}(f, 1-f)=\{\widehat{f, 1-f}\}_{2} \text { for } f \neq 1 \text {. }
$$

Moreover, since $\{\widetilde{f,-f}\}_{2}=1$, the condition $c_{2}(f,-f)=1$ must be satisfied and it follows from the equality

$$
\widetilde{\{f, g\}_{2}}=\widetilde{\{g, f\}_{2}}
$$

that $c_{2}$ must be a symmetric 2-cocycle.

Furthermore, since $c_{2}\left(f^{2}, g\right)=c_{2}\left(f, g^{2}\right)=1$, we have that $c_{2}$ is characterized by its values in $-1,2$ and 5 , which are the generators of the group $\mathbb{Q}_{2}^{*} /\left(\mathbb{Q}_{2}^{*}\right)^{2}$.

Bearing in mind the previous considerations, we have that

$$
\left.c_{2}(2,-1)=\widehat{\{2,-1}\right\}_{2}=1, \quad c_{2}(2,2)=c_{2}(2,-2) c_{2}(2,-1)=1 .
$$


From the equalities

$$
\left.c_{2}(5,-4)=\widetilde{\{5,-4}\right\}_{2}=1 \text { and } c_{2}\left(5,2^{2}\right)=1 \text {, }
$$

we deduce that $c_{2}(5,-1)=1$. It is now clear that $c_{2}(5,5)=1$.

To conclude, let us first assume that $c_{2}(-1,-1)=1$. Then, since $\epsilon(3)=1$ and $\omega(3)=1$ we have that $3=-5 u^{2}$. Moreover, $c_{2}(6,-5)=\{6,-5\}_{2}=-1$, and thus

$$
\begin{aligned}
c_{2}(2,5) & =c_{2}(2,-5)=-c_{2}(12,-5)=-c_{2}(3,-5) \\
& =-c_{2}(-5,-5)=-c_{2}(-1,-1)=-1 .
\end{aligned}
$$

Hence if we write $f=(-1)^{\epsilon(u)} 2^{\alpha} 5^{\omega(u)} \bar{u}^{2}$ and $g=(-1)^{\epsilon(v)} 2^{\beta} 5^{\omega(v)} \bar{v}^{2}$, we have in this case that

$$
c_{2}(f, g)=c_{2}\left(2^{\alpha}, 5^{\omega(v)}\right) c_{2}\left(5^{\omega(u)}, 2^{\beta}\right)=(-1)^{\alpha \omega(v)+\beta \omega(u)}=\widetilde{\{f, g\}_{2}}
$$

and $v=c_{2}[\widetilde{r, \cdot}\}_{2}=1$ is the trivial Steinberg symbol.

Finally, when $c_{2}(-1,-1)=-1$ we deduce, using a similar argument, that $c_{2}(2,5)=1$. Hence, $c_{2}(f, g)=(-1)^{\epsilon(u) \epsilon(v)}$, and

$$
v(f, g)=(-1)^{\epsilon(u) \epsilon(v)} \widetilde{\{f, g\}_{2}}=(f, g)_{2} .
$$

Furthermore, since $c_{2}(-1,-1) \neq 1$ we have that $c_{2}$ is not a 2 -coboundary and we conclude the proof.

\section{Acknowledgements}

The author wishes to thank Professors José Mourao, Joao Nunes and Carlos Florentino at the Instituto Superior Técnico of Lisbon (Portugal) for their hospitality during a three-month period in 2001 when much of the work of this paper was done.

\section{References}

[1] E. Arbarello, C. de Concini and V. G. Kac, 'The infinite wedge representation and the reciprocity law for algebraic curves', in: Theta functions - Bowdoin 1987 (Brunswick, ME. 1987), Proc. Sympos. Pure Math. 49, Part I (Amer. Math. Soc., Providence, RI, 1989) pp. 171-190.

[2] J. Milnor, Introduction to algebraic K-theory, Ann. of Math. Stud. 72 (Princeton University Press, Princeton, 1971).

[3] F. Pablos Romo, 'On the tame symbol of an algebraic curve', Comm. Algebra 30 (2002), 4349-4368.

[4] __. 'Algebraic construction of the tame symbol and the Parshin symbol on a surface', J. Algebra 274 (2004), 335-346. 
[5] J. P. Serre, Groupes algébriques et corps de classes (Hermann, Paris, 1959).

[6] - A course in arithmetic (Springer, New York, 1973).

[7] J. Tate, 'Residues of differentials on curves', Ann. Sci. École Norm. Sup. 4 (1968), 149-159.

Departamento de Matemáticas

Universidad de Salamanca

Plaza de la Merced 1-4

37008 Salamanca

Spain

e-mail: fpablos@usal.es 